\title{
UN VASO DE VIDRIO TARDOHELENÍSTICO PROCEDENTE DEL YACIMIENTO DE BIBRACTE, MONT BEUVRAY, BORGOÑA
}

\author{
ALMUDENA DOMÍNGUEZ ARRANZ1 \\ Universidad de Zaragoza \\ ESPERANZA ORTIZ PALOMAR
}

\begin{abstract}
Bibracte, en el macizo del Morvan, fue la efímera capital de los Eduos. Acogió durante la segunda Edad del Hierro un asentamiento que desempeñó un papel de vital importancia en la guerra de las Galias. Varios programas de investigación han proporcio.zado información relevante sobre el urbanismo al interior del doble recinto amurallado del tipo más tradicional de murus gallicus. El material mueble recuperado ha permitido ilustrar el alto nivel de vida cotidiana en el lugar antes de su abandono y su traslado a Augustodunum. Entre los testimonios se cuentan escasos objetos de tocador, de adorno y vajilla de vidrio. Este es el interés del vaso de vidrio moldeado y monocromo que analizamos tanto desde de los orígenes de este tipo de objeto como desde sus aspectos de fabricación. Las características técnicas de la pieza y los testimonios de talleres de fabricantes de vidrio, en el Próximo Oriente, determinan que pudo ser Jerusalén el lugar de producción de este objeto en el siglo I a.C. En esta ciudad se conoce una producción de vidrio moldeado y monocromo, con una difusión a larga distancia incluso hasta el Extremo Occidente donde se señala la presencia de objetos como el que se ha descubierto en Bibracte.
\end{abstract}

Bibracte, dans le massif du Morvan, a été l'éphémère capitale des Éduens. Cet habitat du deuxième âge du Fer a joué un rôle de toute première importance lors de la guerre des Gaules. Plusieurs programmes de recherche ont fourni des informations sur l'urbanisme à l'intérieur d'une double enceinte de murus gallicus. Le mobilier recueilli a permis d'illustrer le haut niveau de la vie quotidienne sur ce site, avant son abandon et son transfert sur le site d'Augustodunum. Parmi les témoignages de la vie quotidienne, on compte de certaines objets de toilette, de parure et des récipients en verre. Ainsi, cette intéressante coupe de verre monochrome moulée présentée ici. Notre étude porte sur les caractéristiques techniques et sur les origines de ce genre d'objet. Les principaux traits de cette pièce et les témoignages d'ateliers mis au jour au Proche-Orient conduisent à placer le lieu de production de cet objet dans la Jérusalem du ler siècle av. J.-C. C'est dans cette ville que l'on connaît des productions avec une diffusion sur de longues distances, jusque dans l'extrême Occident où l'on signale la présence d'autres objets comme celui qui a été découvert à Bibracte.

1. En el siglo XIX J.-G. Bulliot y J. Déchelette hicieron posible el conocimiento científico del yacimiento de Bibracte, en el macizo del Morvan, aunque las referencias en De Bello Gallico habian puesto sobre la pista de la importancia de esta ciudad, que fue capital de los Eduos ${ }^{2}$. De las excavaciones practicadas por entonces se pudo determinar su cronología de la segunda Edad del Hierro con el momento de mayor apogeo en época de César, debido a la política que Vercingetorix, caudillo de los Eduos, adoptó en la sublevación de las Galias tras la derrota de Gergovia. Ya en época de Augusto los habitantes de Bibracte se trasladaron a la llanura, ocupando la nueva fundación de Augustodunum, la actual Autun, llevándose consigo la mayor parte de sus pertenencias como revela la arqueología.

La investigación de Bibracte, quedó paralizada tras la muerte de Déchelette durante la primera guerra mundial, y no se volvió a emprender hasta 1984 cuando el yacimiento se transforma en un centro de investigación internacional y punto de referencia para los estudios célticos, el Centre archéologique eu-

\footnotetext{
1 Profesora Titular de Arqueología, Epigrafía y Numismática de la Universidad de Zaragoza. E-mail: aldomin@unizar.es

2 Para las excavaciones anteriores al siglo $X X$ puede consultarse J.-G. Bulliot (1899) y J. Déchelette (1903 y 1904) y obras citadas. Una síntesis sobre la importancia histórica del yacimiento y su entorno en Ch. Goudineau, Ch. Peyre (1993).
} 


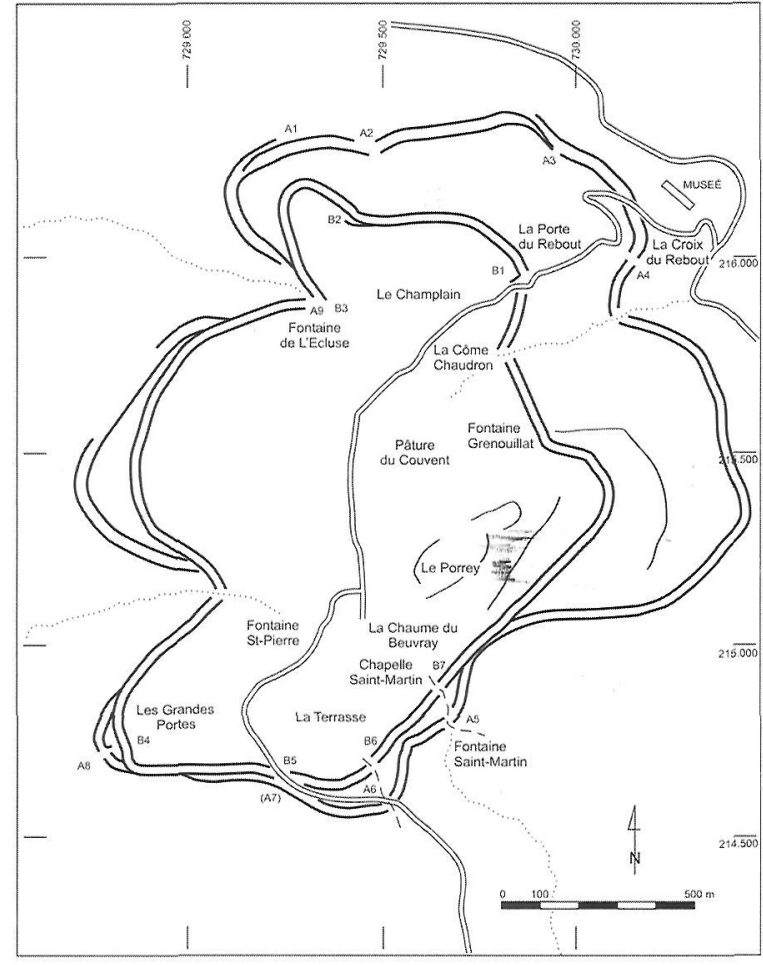

Figura 1: Plano de situación de los distintos sectores del oppidum de Bibracte al interior de las dos murallas que lo rodean.

ropéenne du Mont Beuvray. Del control de los trabajos de campo y de la divulgación científica se encarga un Comité formado por científicos de las instituciones de los países participantes, que son nombrados por el Ministère de la Culture, beneficiándose de esta forma de la colaboración de equipos de diferentes universidades, entre las cuales se cuenta la Universidad de Zaragoza ${ }^{3}$.

La investigación efectuada por la Universidad de Zaragoza en el transcurso de varias campañas de excavación, entre 1994 y 19994, ha llevado al conocimiento de las características de un barrio residencial y de artesanos instalado hacia el siglo I a.C. en la ínsula I, y dos de las principales vías de circulación dentro del oppidum (Figs. 1 y 2). De una parte está la calle de las Bodegas cuyo trayecto es perpendicular al cardo maximus o la calle del Estanque, que discurre de norte a sur entre las dos puertas principales, la Porte de Rebout y Les Grandes Portes. La calle de las Bodegas, en la Pâture du couvent, facilita la comunicación las ínsulas, I y II, las fachadas de cuyos edificios la jalonan respectivamente por el este y por el oeste, y el sector central organizado en torno al estanque monumental con el sector sur del área residencial. De otra parte, se encuentra la calle trasversal a la de las Bodegas, en una posición más meridional, con un papel de comunicación entre la ínsula I y el barrio de artesanos metalúrgicos de la Côme Chaudron; esta calle secundaria sigue en parte el trayecto del camino medieval que ha servido de acceso hasta tiempos modernos para llegar a la parte superior de la ciudad donde se implantó en el siglo XV una comunidad de monjes franciscanos.

2. El descubrimiento del recipiente de vidrio objeto del presente estudio se produjo en el edificio 1 o casa de la fosa de los vasos pintados (Fig. 3), junto a la parte externa del muro oriental que da a la calle de las Bodegas, en el relleno de la trinchera que se abrió para efectuar una remodelación del edificio posterior al momento de sü construcción en torno al 30 a.C.. Hablamos del edificio ubicado en el ángulo más oriental de la ínsula I, en realidad la única construcción erigida sobre cimientos de piedra

${ }^{3}$ Los trabajos actuales por la parte española se llevan a cabo bajo la dirección de A. Domínguez-Arranz y J. Gran-Aymerich, apoyados por tres ayudas del Ministerio de Ciencia y Tecnología español y del Ministerio de Asuntos Exteriores francés a través del programa de Acciones Bilaterales entre los dos países, siendo los Dres. Dominguez Arranz y Gran-Aymerich los investigadores principales respectivamente por España y Francia. Estas Acciones son: 1996: Fenómenos de urbanización en Iberia y la Galia (siglos VI- I a. de C.). DGYCITCNRS (Acción concertada dentro del Programa franco-español de cooperación en Ciencias sociales y Humanas). 19981999: Aspectos de protourbanismo y primer fenómeno urbano en Europa Occidental: análisis de contactos culturales entre el mundo mediterráneo y el continente (Programa Sectorial de Promoción del Conocimiento del Ministerio de Educación y Cultura, España. Acción Integrada Francia-España. HF1997-0043). 2001-2002: Acerca de los origenes de las primeras ciudades: el testimonio de los obsequios diplomáticos (Programa Sectorial de Promoción del Conocimiento del Ministerio de Educación y Cultura, España. Acción Integrada Francia-España. HF2000-0096). También se ha contado durante varios años con el apoyo de la Caja de Ahorros de la Inmaculada.

4 Agradecemos especialmente la colaboración en los trabajos de campo y gabinete de José $\mathrm{M}^{\mathrm{a}}$ Vallejo Rasero, Abigail Pereta Aybar y Alberto Miñón Querejeta, primero en condición de alumnos y posteriormente como arqueólogos y colaboradores científicos, además de los numerosos alumnos que de forma voluntaria han pasado por el yacimiento y dejado su inevitable impronta a lo largo de estos años. El arqueólogo Alfredo Blanco Morte ha prestado una ayuda imprescindible en la elaboración de los dibujos planimétricos y altimetrías, no limitándose a su elaboración técnica sino también aportando valiosas sugerencias a la interpretación de la topografía del sector, así como también en relación con el diseño definitivo de los diversos materiales muebles descubiertos, junto con Sonia Montanel Tramullas, Sonia Ruíz Llera y Patricia Pérez Dios. 


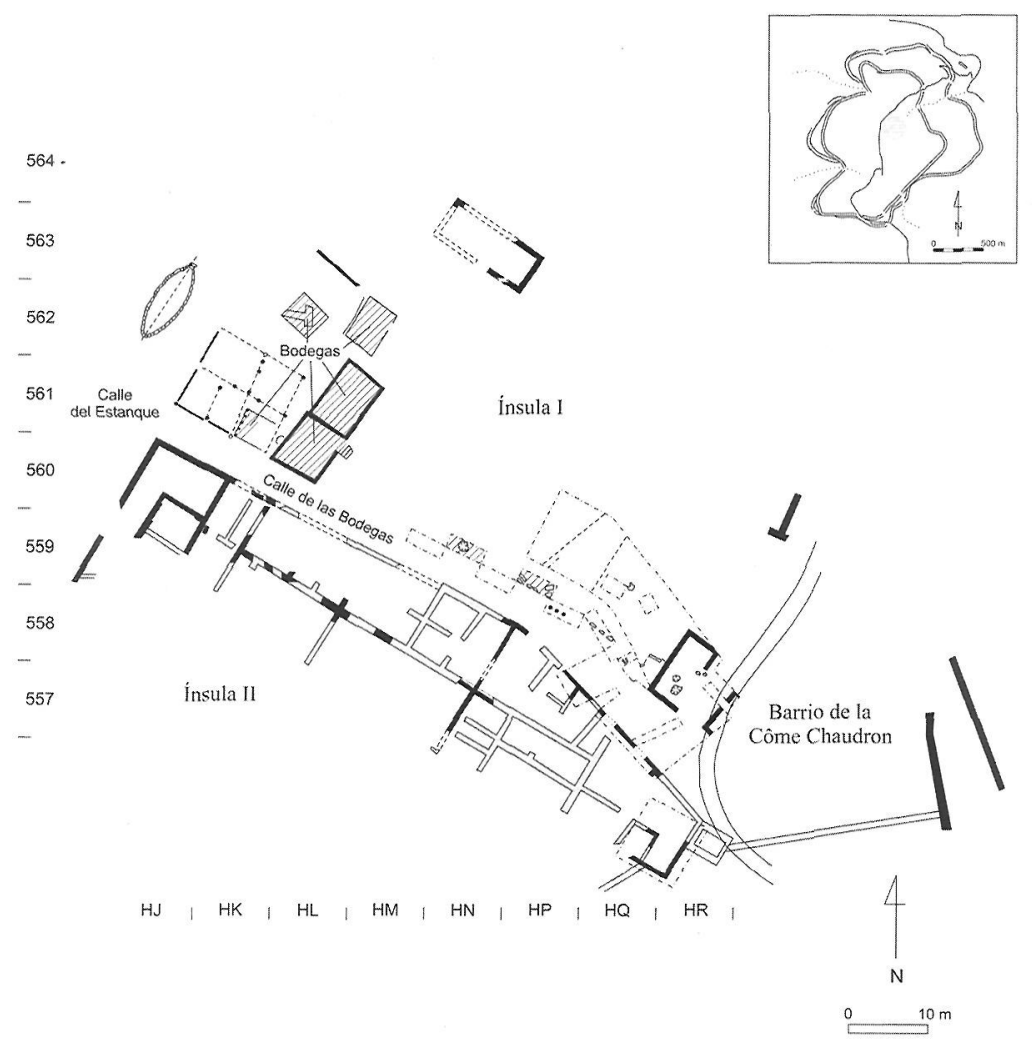

Figura 2: El urbanismo de la "Pâture du Couvent": los sectores excavados de la insula I (ínsula de las Bodegas) y de la ínsula Il (ínsula de las Forjas) y las vías de comunicación reconocidas.

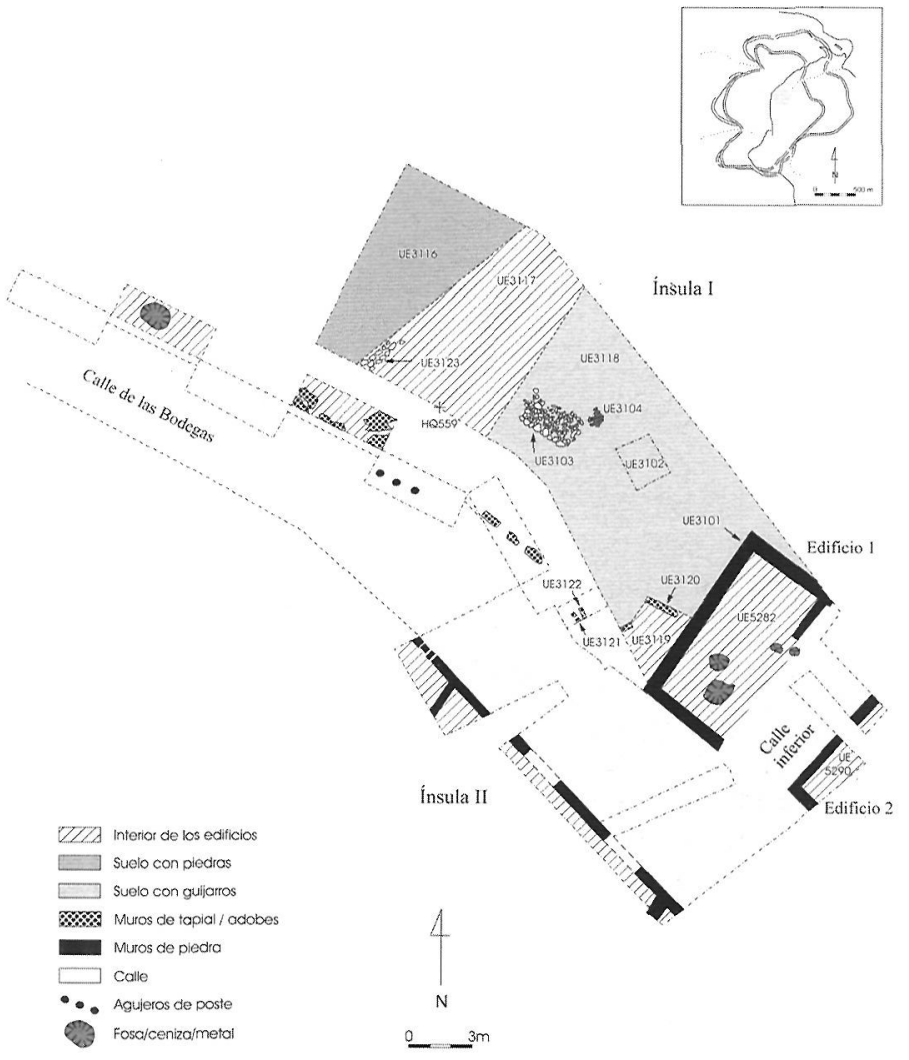

Figura 3: Situación de la casa de la fosa de los vasos pintados (edificio 1) en la ínsula I. 
descubierta en este sector inferior de la ínsula, que cuenta con una superficie de casi $50 \mathrm{~m}^{2}$.

A primera vista podria parecer una casa con bodega de la misma apariencia y tipología de las constatadas en otros sectores del oppidum $^{5}$ (ver Fig. 2, sector noroeste), sin embargo tras su excavación esta apreciación se descartó inmediatamente interpretándose como una casa-taller o taller-tienda probablemente de artesanos orfebres, con la fachada principal orientada hacia la calle que separa esta ínsula de los edificios de la Côme Chaudron (Domínguez y Gran-Aymerich, 2001, 163-164). El interior de la construcción ha estado probablemente dividido en dos espacios a juzgar por la diferencia de niveles de suelo entre la parte norte y la parte sur, y los vestigios de actividades artesanales: el fondo de una fosa interpretada como la base de un gran hogar, una fosa-cenicero y una fuerte concentración de escorias y desechos de hierro, clavos o útiles de hierro probablemente ligados a una actividad de transformación de objetos de hierro. Estos vestigios se concentran en la parte meridional en tanto que la mitad norte no ha aportado ninguna evidencia de trabajo, lo que sugiere la existencia de un espacio con acceso directo a la calle, determinado por una interrupción de la fachada oriental y la impronta de dos postes.

En el interior de la ínsula, el tipo de vivienda característico es de tradición gala con utilización masiva de las rocas autóctonas y materias primas disponibles en el entorno. Salvo en los ángulos occidental y oriental no hay testimonios del empleo de muros de piedra ni siquiera para construir los basamentos, por lo que con toda probabilidad la arcilla fue el elemento de base de los muros reforzados con postes de madera verticales, la gravilla apisonada para el acondicionamiento de los espacios exteriores y callejones de acceso a las áreas interiores de la ínsula, y la arcilla mezclada con gravilla más fina o simplemente apisonada en los pavimentos interiores de las casas.

Al igual que en la parte superior más próxima al Estanque ${ }^{6}$ los tramos inferior e intermedio de la insula de las Bodegas han revelado la existencia de amplias superficies de habitación o áreas destinadas a actividades artesanales, con una gran presencia de carbones, de tierras quemadas, zonas de extensión de cenizas, escorias de hierro y abundante mobiliar cerámico. La ausencia de muros sóli- dos o basamentos de piedra y en algunos puntos la acumulación de bloques de adobe o de tapial formando parte del nivel de abandono, además de varios indicios de improntas de postes, lleva a pensar que se trata efectivamente de superficies a cielo abierto que alternan con construcciones ligeras cubiertas a su vez de techumbres soportadas por postes de madera. En este sector se produjo un excepcional hallazgo, un entalle de anillo de ágata traslúcida con un personaje grabado, además de varias monedas galas de bronce.

Por otra parte entre el cardo maximus y la calle inferior, que discurre en dirección noreste y separa la ínsula I del barrio de la Côme Chaudron (Fig. 2), no se han identificado por el momento otras calles secundarias, de forma que es presumible que el acceso al interior de la ínsula I estuviera resuelto a través de espacios internos o patios descubiertos, como se deduce de las excavaciones practicadas sobre un amplio sector?.

En lo que concierne a los espacios interiores de la ínsula, hay que señalar en general la fragilidad de los restos que corresponden a las construcciones de las viviendas (Fig. 3), lo que contrasta con lo que ocurre en la insula II donde es patente la mejor conservación de las construcciones por la intervención de materiales de mayor entidad: basas y elementos de fustes de columnas o de claves de arcos en piedra caliza, losas y piedras talladas, fragmen-

\footnotetext{
${ }^{5}$ Estas bodegas, construidas en madera y en piedra, fueron excavadas por los equipos de la Universidad Complutense de Madrid, dirigidos por los Dres. Martín Almagro, Jean Gran Aymerich y Francisca Hernández, y de la Universidad Libre de Bruselas, bajo la dirección del Dr. P. P. Bonenfant, en el ángulo occidental de la ínsula I delimitado por la calle del Estanque y la calle de las Bodegas, y en otros lugares del yacimiento. Actualmente se ha reconstruido una de estas bodegas en el lado oeste de la calle del Estanque.

${ }^{6}$ Las investigaciones de la parte meridional de la insula y del Estanque fueron emprendidas por un equipo la Universidad Complutense y el Centre National de la Recherche Scientifique (CNRS) entre 1987 y 1992. La principal publicación que reúne toda la información arqueológica y relación de hallazgos en este ámbito es: M. Almagro-Gorbea y J. Gran-Aymerich (1991) con una amplia bibliografía de referencia, además de los sucesivos informes publicados por el CAE al término de cada campaña. Actualmente está pendiente la publicación de la memoria final de las excavaciones.

${ }^{7}$ Los sondeos practicados en el sector intermedio de la ínsula pusieron al descubierto suelos que se identificaron con áreas cubiertas y otras descubiertas, estas últimas podrian haber funcionado como patios o espacios de acceso a las viviendas o talleres: A. Dominguez Arranz y J. Gran-Aymerich (1996, 2-26).
} 
tos de tejas planas e ímbrices que indican la naturaleza de la techumbre, algunas de las cuales presentan trazas de estuco coloreado en ocre rojo, además de fragmentos de terracotas arquitectónicos.

\section{Por todo el sector se han indivi-} dualizado materiales residuales del primer período de ocupación, el repertorio mobiliar más antiguo en este sector corresponde a la segunda mitad de La Tène D1, entre fines del siglo II y principios del I a.C.. Entre el material fragmentario destaca un conjunto de vasijas, algunas completas, de superficie pulida o engobada y pintada con motivos geométricos o motivos florales, que fue hallado reunido en una fosa bajo el suelo de la casa y próxima al umbral ${ }^{8}$. Es la única estructura que conocemos de este período en el sector.

La fase cronológica mejor documentada es la que corresponde al período posterior a la conquista y primeros años del reinado de Augusto, a la cual se atribuye una gran cantidad de fragmentos de ánforas, la mayoría itálicas republicanas (algunos fragmentos con estampillas) en asociación con otros materiales importados o bien fabricados en talleres locales ${ }^{9}$

La cerámica de importación está representada por vajilla de tipo campaniense, sigillata y de paredes finas. En cerámica campaniense sobresalen los cuencos Lamb. 1 y las páteras Lamb. 5 ó 7 . Sin embargo las producciones aretinas y de paredes finas son las que contribuyen a concretar mejor la cronología de las estructuras edilicias entre el 30 a.C. hasta 15-20 de C.. Así, las formas aretinas abiertas, atribuidas a tipologías Consp.1.1. y 11.1. y Consp.14, principalmente, y formas cerradas, casi todas asimiladas a la tipología Consp.50. Algunas conservan sellos radiales y centrales (a nombre de Sestius Dama, P. Hertorius, Ateius, A. Sestius Ampio, P. Attius), de gran valor para la datación.

Los productos de paredes finas corresponden a importaciones, de procedencia gálica o itálica, y también imitaciones de fabricación local. Generalmente las piezas más antiguas carecen de engobe el cual se va imponiendo desde el periodo augusteo. Hay decoraciones que son imitaciones de palmetas estampadas, ornamentación conocida como "tipo Beuvray" por su frecuencia en las cerámicas del yacimiento. Las importadas presentan engobe y decoración variada, a base de motivos vegetales y decoración cordada a la barbotina y otros hechos con ruedecilla. Estaríamos ante vajilla importada fechada por el engobe y la decoración en el período augusteo, y producciones indígenas que comienzan a extenderse alrededor del 30 a.C. y perduran en el primer decenio del I de C.

En el conjunto es lógica la presencia de cerámicas de fabricación local de diversas categorías y acabados según su destino y función: escudillas, cuencos, botellas, jarras, de buen acabado, consideradas vajilla fina de mesa; tinajas de diferentes tamaños para el almacenaje de alimentos; o piezas de vajilla destinadas a usos culinarios, como ollas, potes, cazuelas, de factura más grosera y cocción reductora, a veces con un revestimiento micáceo singular o más frecuentemente con una cubierta orgánica negra.

No faltan los utensilios empleados por los artesanos cuya materia prima por excelencia era el cobre, hierro y plomo. Quedan los propios instrumentos, el material de desecho, fragmentos de lingotes, y algunos de los productos finales, también de metal, que fueron utilizados en la construcción o formaron parte del mobiliario (clavos, remaches, elementos decorativos). Pocos objetos quedaron en relación con las actividades domésticas (pesos, anzuelos, llaves, pasadores) y el atuendo personal (hebillas, fíbulas) y el relacionado con el soldado (clavos de sandalia, paragnathides, regatón de lanza, manípulo de escudo). Varias monedas galas de plata, bronce y hierro y otras romanas de Nemausus (Nimes) fueron halladas repartidas en la estratigrafía de las vías de

\footnotetext{
${ }^{8}$ El hallazgo fue publicado en A. Dominguez Arranz y J. GranAymerich (1998, 97-106); hay una breve referencia a este descubrimiento en L'Archéologue, $n^{\circ} 43$, août-septembre 1999, 13. La vasija más notable está expuesta en el Museo de la civilización céltica de Bibracte, de la que se puede contemplar una reproducción en Les celtes et les arts du feu. Armes, outils, bijoux. Dossiers d'Archéologie, $n^{\circ} 258$, nov. 2000, p. 36: un vaso alto pintado con decoración moteada y motivos solares, realizada con una técnica típica de los talleres del Centro-Este de la Galia, desde el Morvan hasta el valle medio del Rhin, que consiste en plantear la decoración en negativo con cera o grasa y sobre ella aplicar un pigmento de color. La funcionalidad de la fosa y el material cerámico se encuentra actualmente en estudio por parte de Almudena Domínguez Arranz y Patricia Pérez Dios.

${ }^{9}$ Vean un avance de la tipología y cronología de los materiales arqueológicos en A. Domínguez Arranz y J. Gran-Aymerich (2004, en prensa). El estudio de las ánforas y los sellos, que aportan una valiosa información sobre las procedencias y cronología de estos envases, en A. Dominguez-Arranz, J.M. Vallejo Rasero, S. Ruíz Llera, (2004, 61-70). Por lo que respecta al material numismático, muy útil para establecer la cronología de los estratos arqueológicos, una aproximación en A. Domínguez-Arranz, A. Miñón Querejeta (2003, 47-54).
} 


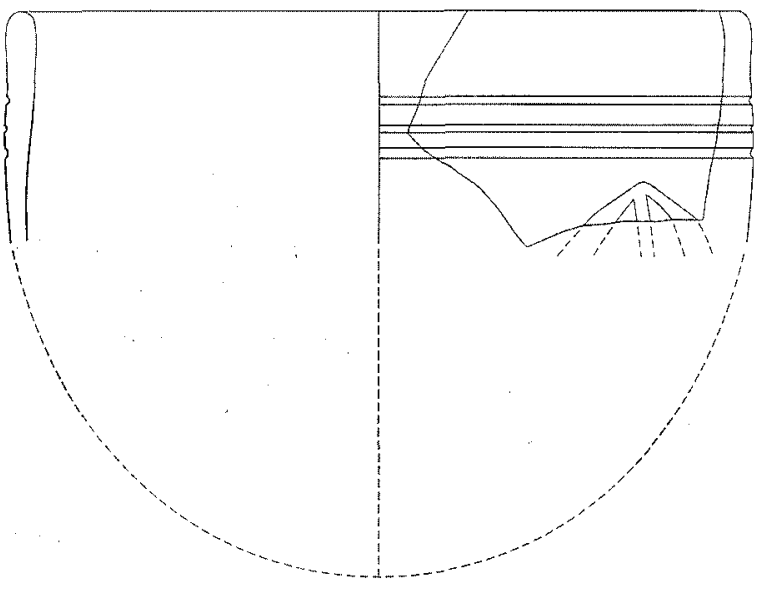

0 $5 \mathrm{~cm}$.

Figura 4: Vaso de vidrio hallado en el sector. Propuesta de reconstrucción tipológica. (Dibujo: A. Blanco).

circulación y en el interior del edificio, lo que confirma su uso en los intercambios comerciales.

Los elementos de adorno fabricados en otras materias apenas se pueden certificar, en pasta vitrea (colgantes) y en piedras semipreciosas (entalle de ágata). Excepcionalmente se conserva una terracota fragmentada, quizás parte de una figurilla humana modelada.

4. Es un hecho que la fragilidad del vidrio no hace buena su conservación en los yacimientos arqueológicos, en éste se suma el hecho del abandono del lugar para ocupar otro asentamiento en la llanura, llevándose sus habitantes la mayor parte de sus pertenencias, y sobre todo aquellas más valoradas como podían ser las piezas de vidrio, de ahí el interés de la que se estudia. El vidrio recuperado en otros sectores del yacimiento, de los períodos medieval y moderno, procede en buena parte de las proximidades al convento de franciscanos erigido sobre los fundamentos de los edificios de época augustea en la ínsula II. EI vidrio fue sin duda utilizado tanto en la vajilla de lujo como en la fabricación de objetos rituales, piezas finas de tocador y objetos de adorno. Excepcionalmente en la excavación del Estanque monumental, en la vía principal del oppidum, aparecieron dos fragmentos de brazaletes típicos celtas de pasta vítrea, uno de color violeta y sección triangular, y el otro de color azul y sección lobulada con decoración geométrica ${ }^{10}$. El sector excavado por la Universidad de Zaragoza proporcionó escasos y pequeños fragmentos de vidrio de formas indeterminadas, una anilla de pasta vitrea traslúcida, de sección circular, posiblemente de un colgante y un vaso de vidrio monócromo con decoración tallada en frío de temática vegetal. Vamos a centrarnos precisamente en el estudio de este recipiente por ser una pieza poco común (Fig. 4), fabricada con una materia prima considerada de prestigio, por ello convertida en objeto apreciado y trasmitido de generación en generación. Interesa destacar la singularidad del hallazgo: se encontraba con otros materiales formando parte del relleno de una trinchera que fue abierta junto al muro oeste de la casa mencionada, en el ángulo oriental de la ínsula, al efectuar una reforma de este muro en el último período de ocupación. Desgraciadamente la trinchera practicada a causa de esta remodelación truncó la estratigrafía original de contacto de la calle con el muro de la edificación aunque a su vez ha permitido constatar esta última fase.

4.1. Atendiendo al perfil, características del vidrio, diseño y modalidad decorativa, así como rasgos tecnológicos de la manufactura puede determinarse que este fragmento de vidrio corresponde a los cuadros constituidos por variantes de vidrios moldeados monocromos con estrías y con decoración vegetal, de Nenna, tipos: Fig. 3.d (Fig. 5.2) y Fig. 9. a (Fig. 5.1), respectivamente ${ }^{11}$. Siendo el objeto que analizamos, desde el punto de vista compositivo, una mezcla de estrías y dibujos vegetales. Las piezas con decoración vegetal no hacen sino reproducir perfiles ya conocidos entre las vasijas decoradas con ranuras. Los resultados de combinar número de estrías, proximidad, perfil, color, etc. son muy numerosos. El trabajo en vidrio por unos aspectos $u$ otros de su manufactura hace que cada realización sea única resultando muy difícil ver copias idénticas.

El desarrollo del perfil en forma de casquete esférico es en realidad un precedente de la forma Isings 1, que puede considerarse un arquetipo (Isings, 1957, 15-17).

La descripción del galbo responde a un borde recto y pulido en el canto cuya pared va adoptando la forma de un huso progresivamente y acaba en un fondo convexo. Diámetros, dimensiones globales y declinación de las pa-

\footnotetext{
${ }^{10}$ Estudiados en: M. Almagro-Gorbea y J. Gran-Aymerich, (1991), vean especialmente las páginas 143-144.

${ }^{11}$ Vean M. D. Nenna (1993, 11-21), se acompaña a nuestro texto una figura tomada de esta publicación donde pueden observarse las diferentes variantes constituidas por el autor en relación con la presente tipología de vidrio.
} 

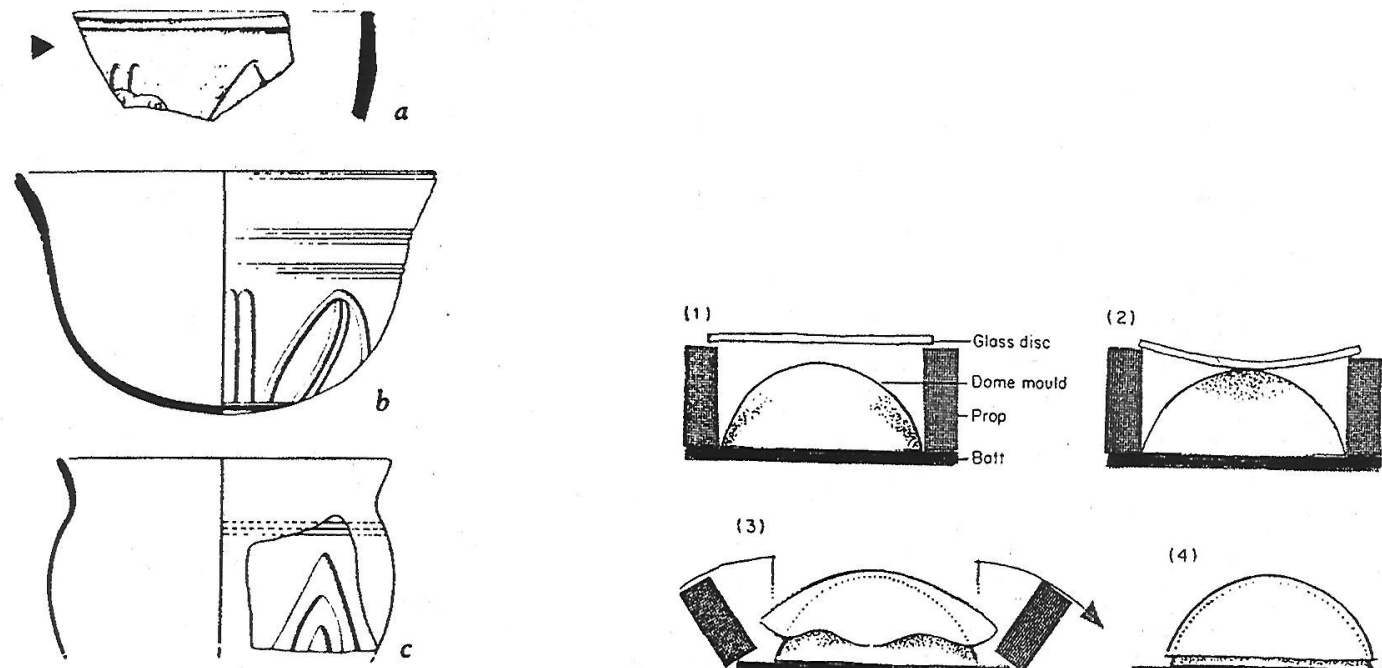

(3)
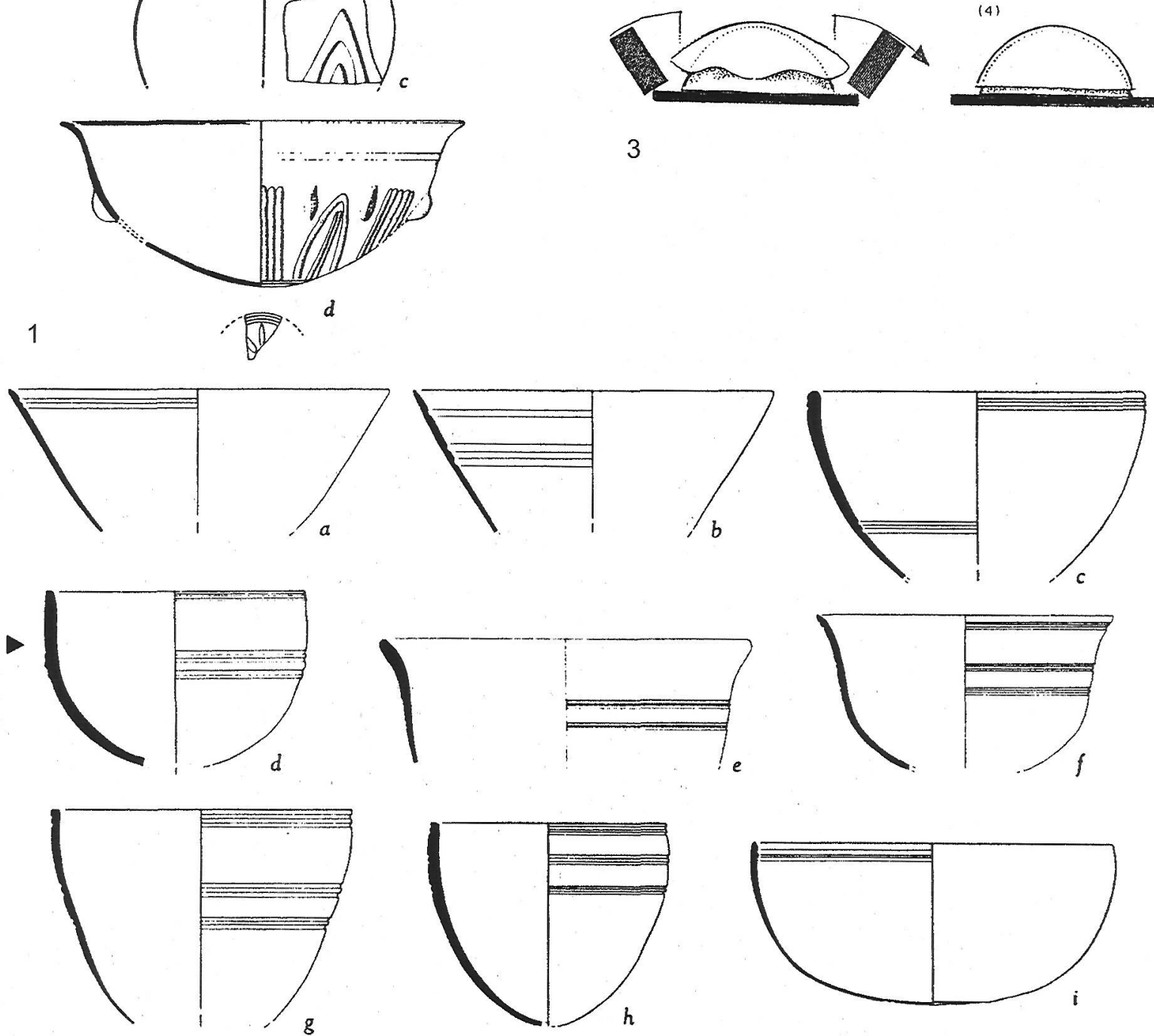

2
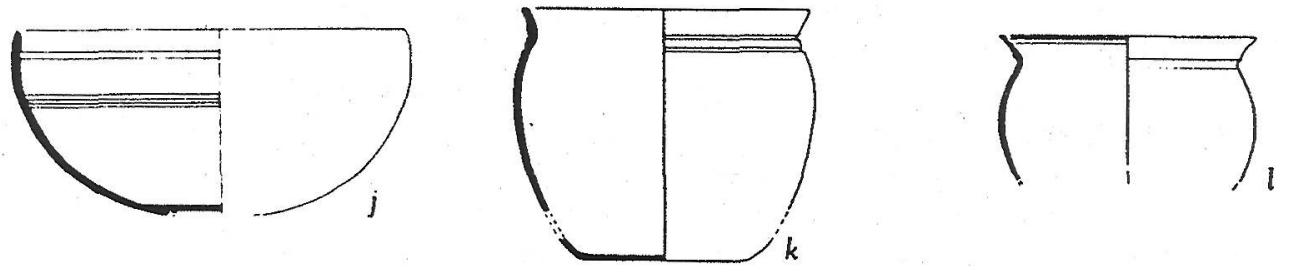

Figura 5: Vidrio helenístico. 1- Variantes de vidrios modelados monocromos, con decoración vegetal (Nenna, 1993, 18, Fig. 9). 2- Variantes de vidrios modelados monocromos, con decoración de ranuras (Nenna, 1993, 14, Fig. 3). 3- Proceso de fabricación «modelado sobre horma» partiendo de un «blanco» de vidrio; tomado de (Sternini, 1995, 100, Fig. 153). 
redes pueden variar considerablemente; además se fabricaron perfiles con los bordes exvasados. Los vidrios de esta serie son singulares por su consistencia.

Estamos, pues, ante una forma abierta, de uso doméstico, integrada en la vajilla de mesa. De su posible uso mixto se desprende que pudo emplearse para consumir líquidos o semilíquidos. De acuerdo a sus dimensiones y al coeficiente deducible de la relación abertura-altura, acogiéndonos a la normativa reproducida por Bats, estamos ante un vaso (Bats, 1988, 23-25). Nosotros puntualizamos, que dada la calidad de la pieza y el contexto histórico al que pertenece es muy probable que se utilizara, preferentemente, para beber y uso individual. Obedece al grupo de funcionalidad: 1.1.2.1., según la clasificación establecida por E. Ortiz Palomar (Ortiz Palomar, 2001, 94-95).

4.2. El método de ejecución se denomina «modelado sobre horma». Se trata, junto con la fusión dentro de molde o matriz, de una técnica que precedió al soplado del vidrio, que fue inventado en época helenística. De esa manera se fabricaron vasos, cuencos, escudillas y platos. El perfil respondía al concepto más simple de recipiente ciñéndose a un casquete globular que podía variar entre hemisférico, ultrahemisférico o infrahemisférico. En función del vidrio utilizado se contemplan dos tipos: en vidrio monocromo y vidrio policromo (el segundo con modelos de "cintas», «millefiori» y «mosaico»). El proceso de elaboración se desarrollaría de la siguiente manera (Fig. 5.3):

Previamente se procedía a realizar un disco de vidrio, también llamado pastilla o «blanco» en terminología vidriera. Hasta hace pocos años se pensaba que se hacia derramando, sobre un plano de mármol, el vidrio fundido, pero más recientemente se ha observado que esto no sería posible ya que aquél tendería a deslizarse dando un disco de espesor demasiado delgado. Por tanto se supone un estado en el que el disco plano se obtendría partiendo de una masa simple de vidrio puesta a calentar pero nunca de calibre fino en la fusión. Por efecto del calor el vidrio se dilataría formando una pastilla de circunferencia bastante regular y del espesor necesario. Una bóveda dejaba correr el aire para enfriar y por consiguiente también endurecía el vidrio.

a) El vidrio preformado resultante se apoyaba sobre dos puntales en suspensión por encima de la forma en «cuenco» (vaso, es- cudilla o plato hondo) que recibía el nombre de molde formador colocado hacia abajo.

b) En esta posición se calentaba a temperatura controlada para volverlo dúctil sin llegar a fundir, de modo que entraba en contacto con el molde matriz.

c) A la primera señal de ablandamiento, que mostraba el vidrio en estado dúctil, los sostenes eran retirados.

d) El disco comenzaba suavemente a acomodarse al molde abrazando el mismo perfil, al combarse bajo su propio peso.

Ulteriormente era necesario un trabajo de pulido solo al interior del recipiente y sobre el borde, porque la pared exterior estaba ya alisada por el calor. Adicionalmente se procedía a ornamentar el objeto. La decoración tallada en frío fue muy habitual, principalmente en el diseño de estrías paralelas horizontales debajo del borde.

4.3. Dos tipos de ornamentación adoptaron los vidrios modelados sobre horma, la técnica decorativo-formativa a molde, en la que el moldeado podía usarse para decorar los propios vidrios al mismo tiempo que se conformaba el recipiente; y el tallado en frío por medio de una rueda esmeril y herramientas similares a las utilizadas por los grabadores de piedras duras (gemas, cristal de roca, etc.) método al que pertenece el ejemplar que estudiamos y al que nos referiremos únicamente.

La decoración tallada en frio como puede presuponerse se ejecutaba en la fase última de manufactura, una vez que el vidrio había sido conformado y sometido al proceso de enfriamiento gradual o destemplado. Esta fase era de vital importancia para evitar resquebrajamientos o alteraciones indeseables que pudieran incrementar la frágil naturaleza que ya tiene el vidrio de manera innata.

Lo más habitual eran las estrías y acanalados horizontales; variando en profundidad de talla (desde líneas finas esmeriladas hasta incisiones más rebajadas), número, proximidad (llegando a constituir grupos o series), ubicación al interior o al exterior (en el último caso a lo largo de todo el perfil, cuyos límites serian seguidos del borde y cerca de la base, predominando en la mitad superior del recipiente). Estas bandas paralelas son una reminiscencia de las vetas y texturas vistas en los cuencos simples de madera.

Menos común es encontrar una temática geométrica y vegetal aunque siempre de com- 
posiciones y diseños simples; que se combinaban generalmente con las estrías, comentadas anteriormente. Los adornos de mayor destreza se trazan en la cara externa del recipiente. Las piezas con estas decoraciones mistificadas tendrían más valor que las que portaban únicamente ranuras e implicaría, la presencia de un grabador en el seno de los talleres de vidrio o en otros independientes. En el fragmento que estudiamos aquí hemos identificado dicha decoración vegetal. Se distingue por un desarrollo extremadamente simple y homogéneo y se despliega en cuatro perfiles (Fig. 5.1): hemisférico profundo con el borde recto «a», hemisférico profundo con el borde exvasado «b», cuenco profundo con el borde cóncavo «c» y escudilla con borde exvasado "d»; siendo la primera variante a la que se asimilaría el vidrio de Bibracte. En cuanto al tema de la composición se trata de largas hojas apuntadas en su vértice, dotadas de una nervadura central, o dos y raramente nervaduras oblicuas, alternando con haces de juncos. En algunos ejemplares, la decoración está únicamente constituida por hojas. En el fragmento de Bibracte, solo se puede ver la parte superior de una hoja con una nervadura central, desconociendo el resto de la composición, pero deducimos que constaría de los elementos que acabamos de describir.

La mayor parte de las decoraciones (talladas, moldeadas en relieve, pellizcadas, aplicadas, rebajadas, etc.) en vidrio no han de verse como meras manifestaciones artísticas, además tuvieron una función práctica, escasamente tenida en cuenta, de facilitar mejor su agarre, evitando el deslizamiento de los dedos de unas superficies demasiado satinadas y resbaladizas al contacto con los líquidos, el sudor de las propias manos, etc.

En cuanto al color, se distinguen varios modelos: decolorado, marrón dorado (ocre), amarillo-verdoso y verdeamarillento; este último es con el que se identifica esta vasija. En la tabla de color Caran d'Ache se situaría entre el núm. 225 (verde musgo) y el 245 (oliva claro). La calidad es translúcida.

4.4. Estamos ante un producto de vajilla de vidrio manufacturado durante la baja época helenística. Entre los ejemplares con decoración vegetal se pueden definir cuatro formas de cuencos que deben de datarse desde fines del siglo II y comienzos del siglo I a. C. a partir de los contextos conocidos (Grose, 1989, 193194).

Para los recipientes de vidrio que precedieron a la técnica del soplado, que favoreció producciones menos costosas y más asequibles (exceptuando las consideradas de lujo), hay que tener presente el fenómeno de las «herencias de familia». Bajo esa denominación se alude a una serie de elementos residuales que suelen acompañar muchas veces al conjunto de materiales coetáneos con la secuencia histórica que se excava. La transmisión en el tiempo de determinados objetos, especialmente apreciados por su belleza y singularidad, así como por los lazos afectivos establecidos entre sus propietarios, fue algo corriente y admitido. Isings recuerda este hecho, básico para asignar cronologías de manera correcta a determinadas piezas (Isings, 1957, 15-17). Afecta especialmente a objetos de vidrio que por su escasez o calidad artística, al no ser utilizados cotidianamente entre la vajilla de mesa o por ser piezas que aunque se empleaban fueron cuidadosamente manipuladas y aunando una óptima fabricación de mayor consistencia, etc., preservando la vida de ellas. Por consiguiente no es extraño que la vajilla de vidrio se mencionara en la ley romana, porque pasaba de una generación a otra.

Agustín (Sermo, 18, 7), s. IV d. C., hablando de la brevedad de la vida humana se pregunta: «¿No somos más débiles que si fuéramos de vidrio?». Por lo que añade, «incluso si el vidrio es frágil, si tienes cuidado, él perdura un largo tiempo, y encuentras nietos y bisnietos bebiendo de las tazas de sus abuelos y bisabuelos. Tan frágiles objetos han sido preservados a través de los años».

4.5. La mayor concentración ha sido descubierta en la costa siro-palestina. Los principales hallazgos se corresponden con el pecio de Anticythère (70-60 a. C.) y Tell Anafa (Galilea) (fines s. Il-inicios s. I a. C.) (Weinberg, $1970,17-27$ ). Estos dos lugares han permitido especificar bien el repertorio de técnicas, formas y decoraciones de la vajilla en vidrio empleada antes de la invención del vidrio soplado. El material encontrado en Delos ofrece por su abundancia y variedad la posibilidad de aportar a la vez complementos y elementos nuevos al estudio de estas vajillas. El vidrio moldeado monocromo de fines del siglo II y comienzos del siglo I a. C. forma el lote más importante de las piezas recuperadas en Delos y es también esta isla de las Cícladas el primer lugar que ha aportado tantos vidrios helenísticos con decoración vegetal, siendo clave para datar con mayor precisión y distinguir claramente los productos aqueménidas del llamado grupo de Canosa (Nenna, 1993). 
Los vidrios helenísticos con decoración vegetal fueron trabajados en talleres siropalestinos, como los objetos con decoración simple integrada por ranuras. Sin embargo, el cuidado puesto de manifiesto en su decoración hizo, seguramente, incrementar su valor implicando la presencia de grabadores en el seno de los talleres de vidrieros.

A fines del siglo II e inicios del siglo I a. C. no se producian ya en Delos vasijas en vidrio y este último sería importado. Esta situación debió de ser regla general en la mayor parte de las ciudades helenísticas. Atendiendo a las características detectadas sobre evidencias de talleres de fabricantes de vidrio investigados pudo ser Jerusalén, taller de gran importancia con un amplio abanico de productos y no obstante todos en vidrio monocromo, el lugar de procedencia, bastante probable, del recipiente recuperado en Bibracte.

Su distribución alcanza diversos puntos de occidente como ejemplos de Italia, Cartago, Normandía (Sternini, 1995, 100-101).

4.6. El tema de la conservación tiene en el caso que estudiamos un especial interés. Además de valorar el estado de conservación que presenta el vidrio en la actualidad, como una parte integrada en la ficha arqueológica, interpretaremos lo que transmite el vidrio en su valoración inicial. Indudablemente resalta la calidad excepcional, puesta de manifiesto a través de la prácticamente inapreciable presencia de alteraciones sufridas por la acción de los agentes atmosféricos durante el tiempo en el que estuvo enterrado el objeto hasta el momento de ser recuperado, a diferencia de lo que sucede para la mayoría de los vidrios antiguos, de incluso cronologías mucho más recientes. Tampoco se observa ningún tipo de defecto de fabricación, excepto algunas burbujas, lo que pone de manifiesto el cuidado desplegado en la elaboración de unos productos considerados de lujo. A destacar la uniformidad de la pared, con un grosor muy regular; la limpieza, definición del color, la destreza en el tallado.

Sorprenden el gusto y moda cristalizadas en este producto helenístico comparables con algunas piezas tardorromanas (desde la segunda mitad del siglo IV-V) en que se vuelve la vista a colores, técnicas decorativas o diseños inspirados en épocas anteriores y readaptados en el Bajo Imperio, con una tecnología más descuidada y menos depurada en general.

4.7. En sintesis, el ejemplar que estudiamos es un vaso, en forma de casquete esférico,

moldeado a horma, monocromo y con decoración tallada en frío de temática vegetal. Es una producción bien definida estratigráficamente en los niveles de comienzos del s. I a. C., a partir de los contextos de la isla griega de Delos. Tanto en Delos como en Bibracte fue vajilla importada.

La presencia de este vidrio en el Mont Beuvray es muy interesante en varias facetas histórico-arqueológicas. Por un lado corrobora una cronología, para el nivel excavado en el que se halló el material; en segundo lugar, contribuye a completar el mapa de difusión de estas producciones en zonas occidentales del Mediterráneo, sabiendo que su origen arranca del Mediterráneo oriental; en tercer lugar, estamos ante un objeto muy costoso en términos económicos, ilustrando el grado de riqueza de su propietario, si es que fue pertenencia en su contexto y no mero objeto en trayecto de comercio sin destinatario en ese instante.

Su datación en los primeros años del siglo I a. C. concuerda plenamente con el tipo de vidrio y con la ausencia de restos de vidrio soplado, que se origina en Jerusalén hacia el 50 a. C. y se difunde por occidente, desde Italia, en torno al 30/10 a.C. En el sector arqueológico del que procede el hallazgo sólo se ha encontrado este fragmento, condicionando y limitando una visión de perspectiva más amplia para elaborar conclusiones respecto a otros muchos aspectos.

Profa. Almudena Domínguez Dpto. Ciencias de la Antigüedad Facultad de Filosofia y Letras Universidad de Zaragoza 50007 Zaragoza

aldomin@posta.unizar.es

\section{BIBLIOGRAFIA}

AA.VV. 1999: "L'oppidum de Bibracte. Un bilan de onze années de recherches 1984-1995", Gallia, 55, 1-140.

ALMAGRO GORBEA, M. y GRAN AYMERICH, J., 1991: El estanque monumental de Bibracte, Madrid.

BATS, M. 1988: "Vaisselle et alimentation á Olbia de Provence (v. 350-v. 50 av. J.-C.). Modèles culturels et catégories céramiques", Revue d'Archéologie de Narbonnaise, sup. 18, esp. 23-25.

BULLIOT, J. G. 1899: Fouilles du Mont Beuvray (ancienne Bibracte) de 1867 à 1895, t. I, Autun.

CHRISTOL, M., GOUDINEAU, C. 1987-1988: "Nîmes et les Volques Arécomiques au $1^{\text {er }}$ siècle avant J.-C.", Gallia, 45, 87-103

DÉCHELETTE, J. 1903: L'oppidum de Bibracte, Paris-Autun. DÉCHELETTE, J. 1904: Les fouilles du Mont Beuvray de 1897 a 1901, París-Autun.

DOMÍNGUEZ-ARRANZ, A., GRAN-AYMERICH, J. 1994: "La voie secondaire entre l'îlot aux grandes caves et l'îlot du grand atelier de forges", Centre européen d'ar- 
chéologie du Mont Beuvray. Rapport scientifique intermédiaire. Activités 1994, prévisions 1995, 67-77.

DOMINGUEZ-ARRANZ, A., GRAN-AYMERICH, J. 1996: "Modalités du phenomène d'urbanisation. L'urbanisme dans l'oppidum de Bibracte". Document finale de synthèse. Rapport triennal 1993-1995, vol. 1, 2-26.

DOMÍNGUEZ-ARRANZ, A., GRAN-AYMERICH, J. 1997: "Étude du quartier sud-oriental de la Pâture du Couvent (Campagne de fouilles de l'Université de Saragosse et du CNRS)". Centre européen d'archéologie du Mont Beuvray. Rapport scientifique intermédiaire. Activités 1997, prévisions 1998, 115-117 y 123-128, Glux-enGlenne.

DOMÍNGUEZ-ARRANZ, A., GRAN-AYMERICH, J. 1998: Centre européen d'archéologie du Mont Beuvray. Rapport scientifique intermédiaire. Activités 1998, prévisions 1999, 97-106, Glux-en-Glenne.

DOMÍNGUEZ-ARRANZ, A., GRAN-AYMERICH, J. 1999: Centre européen d'archéologie du Mont Beuvray. Rapport scientifique intermédiaire. Activités 1998, prévisions 1999 , 101-112, Glux-en-Glenne.

DOMINGUEZ-ARRANZ, A., GRAN-AYMERICH, J. 2000: Centre européen d'archéologie du Mont Beuvray. Rapport annuel, Glux-en-Glenn.

DOMÍNGUEZ-ARRANZ, A., GRAN-AYMERICH, J. 2003: "Haeduorum oppidum: novedades sobre el urbanismo de Bibracte (Mont Beuvray, Francia)". Colaboradores: J. Ma Vallejo Rasero, A. Miñón Querejeta y S. Ruiz Llera, Ampurias, 54, (en prensa).

DOMINGUEZ-ARRANZ, A., GRAN-AYMERICH, J. 2004: "Bibracte: le secteur de l'angle oriental de l'îlot I à la Pâture du Couvent», Bibracte, 6, (en prensa).

DOMINGUEZ-ARRANZ, A. y MIÑON QUEREJETA, A. 2003:
"Contribución al estudio de los hallazgos monetarios de Mont Beuvray, Borgoña, Francia", Acta numismática, 33 , 47-64.

DOMINGUEZ-ARRANZ, A. y PÉREZ DIOS, P. (e.p.): "La fosa de los vasos pintados en Bibracte. Mont Beuvray, Francia".

DOMÍNGUEZ-ARRANZ, A., VALLEJO, J. M. y RUÍZ, S., 2003: "Las ánforas de Bibracte en la época romana: Tipología y epigrafía", Salduie, 61-70.

GOUDINEAU, C. y PEYRE, C., 1993: Bibracte et les Eduens. À la découverte d'un peuple gaulois, Paris.

GRAN-AYMERICH, J. y DOMÍNGUEZ-ARRANZ, A. 2001 "Bibracte en Borgoña. Un yacimiento arqueológico europeo y catorce años de participación científica española", Trabalhos de Antopologia e Etnologia, 41 (1-2), 157-177.

GRAN AYMERICH, J., 1989: "Les premières phases d'occupation du Mont Beuvray. Données anciennes et recherches en cours", La Civilisation Hallstatt, Rencontre Internationale de Liège, 344-355.

GROSE, D. 1989: The Toledo Museum of Art. Early Ancient Glass, Nueva York.

ISINGS, C. 1957: Roman Glass from Dated Finds, Groningen/Jakarta.

NENNA, M. D. 1993: "La verrerie d'époque hellenistique à Delos", Journal of Glass Studies, 35, 11-21.

ORTIZZ PALOMAR, E. 2001: Vidrios procedentes de la provincia de Zaragoza: El Bajo Imperio Romano. Catálogo: Fondos del Museo de Zaragoza, Zaragoza .

STERNINI, M. 1995: La Fenice di Sabbia. Storia e tecnología del vetro antico, esp. 100-101, Bari.

WEINBERG, G. D. 1970: "Hellenistic Glass from Tell Anafa in upper Galilee", Journal of Glass Studies, 12, 17-27, Corning, Nueva York, 